\title{
viewpoint
}

\section{Before it's too late}

\author{
Why synthetic biologists need an open-parts collaboration—and how to build one
}

\author{
Stephen M. Maurer
}

$\mathrm{F}$ or almost a decade, synthetic biologists have bet their future on the idea that new organisms can be designed by combining short snippets of DNA-socalled standard biological parts-into complex genetic blueprints in the same manner that LINUX modules are now combined to make software. Scientists and their governments are further betting on this vision to create a multi-billion dollar industry; and they seem to be right. Consider, for example, the apparent success of the industry's leading company, Amyris (Emeryville, CA, USA). Four years ago, The Bill \& Melinda Gates Foundation supported the company with US\$50 million to design a new organism that can secrete the precursor of the anti-malarial compound artemisin. Amyris will reportedly achieve this goal on time and under budget, yet this is only the beginning. Although Amyris agreed to do the work at cost, it gained something much more valuable than profit: experience. Having assembled 30 or more parts into a working organism, the company's next project will proceed at a faster pace and cost less money. It has already decided to chase the biggest market of the twenty-first century: biofuels. Amyris claims that its organisms will be turning sugar cane into diesel by 2010, into jet fuel by 2011 and into gasoline by 2013 .

Technologically, then, the idea of assembling standard biological parts into increasingly complex DNA blueprints has worked beautifully. But the standard parts strategy is about more than technology. It also has powerful economic implications. Twenty years ago, the need for standardized software gave us the Microsoft monopoly. Will something similar happen in synthetic biology, or can the community push the analogy in a more hopeful direction; for example, by launching a LINUX-like initiative for standard DNA parts?
The open-parts idea has been around for nearly as long as synthetic biology itself (Cohn, 2005). Its proponents imagine a world in which academic researchers and companies who develop DNA parts share them freely with one other to advance the whole field. This is in contrast to the closedparts strategy, in which developers use patent protection and secrecy to hoard the best parts or otherwise charge others to use them. Unfortunately, the discussion has not made much progress. Open-parts advocates distinguish between parts that 'resemble operating systems' and parts that 'resemble applications programs', and even sometimes parts that become 'devices'. But it is hard to see how such vague distinctions could be used to write a practical license. The open-parts agenda is clearly failing. Plainly, software analogies are not sufficient; to succeed, open-parts pioneers will also need a detailed understanding of the emerging synthetic biology industry and the cold-blooded business reasons that drive sharing.

L et us begin with some basic economics. Suppose that parts A and B are two DNA sequences that perform the same function. In most cases, researchers who inspect the two DNA sequences by eye will not be able to tell which one is better suited to fulfil a given task. Suppose, however, that a company picks part B at random and then spends US\$50 million to make it work together with 30 other parts inside an organism. Now, anyone with access to this experience will know which tricks to use and which pitfalls to avoid in order to make part B work. Although the two parts were originally indistinguishable, projects built around part B are now cheaper and easier than those that use part A. More generally, the value of a part depends not only on its intrinsic cleverness,

\section{Technologically, then, the idea of assembling standard biological parts into increasingly complex DNA blueprints has worked beautifully}

but also on how many times it has been used before (Henkel \& Maurer, 2007).

This is a simple statement. Even so, a great deal of economics flows from it. First, experience is valuable. This means that a part that has been successfully used is more likely to be selected a second and third time. As in the software industry, this leads to a rich-get-richer dynamic, in which small differences in popularity are steadily amplified over time. Put somewhat differently, synthetic biology is a 'tipping market' that is, unstable and prone to monopoly. Second, companies seldom experiment on individual parts, which suggests that this rich-get-richer dynamic will extend to entire constellations of parts. Finally, the tipping dynamic does not care whether the dominant parts constellation is open or closed. If a mature industry springs up around shared parts, so much the better; but companies will also pay for closed parts if necessary. Both solutions are possible and depend on the accidents of history.

This simple model also says something about policy. Patents are supposed to let inventors recover the cost of developing clever new parts. However, the value of a synthetic biology part depends on more than the inventor's initial R\&D investment-it also depends on how many other researchers have subsequently used the part. Since the law allows patent owners to capture both sources of value, this sounds like an unjust windfall. More importantly, society ends up paying high prices without getting anything 


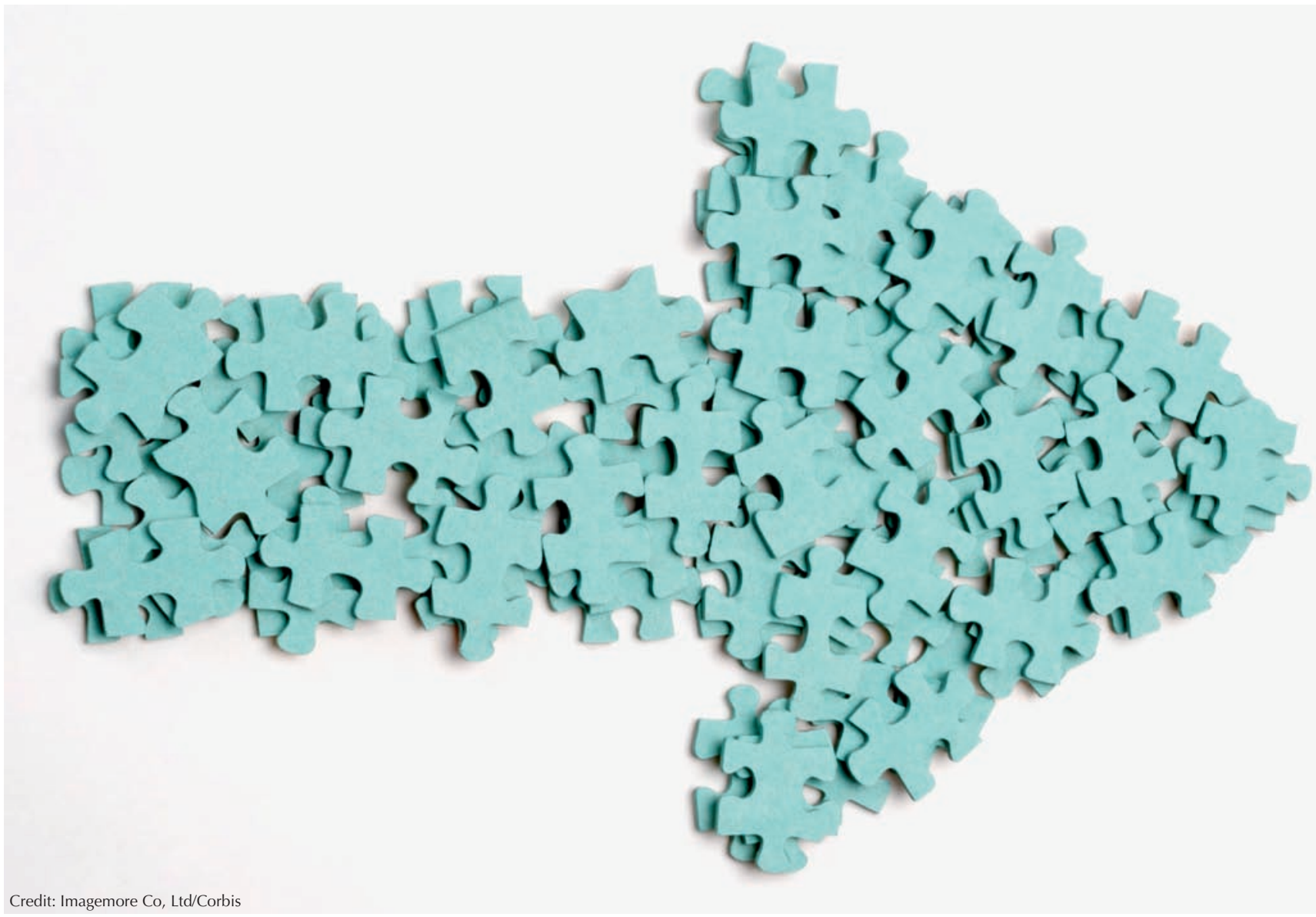

in return. This means that the existence of expensive parts will not matter much to, for example, a patient without insurance or a researcher operating on a shoestring budget, and even affluent users will have less money to spend on other goods. Open parts, which set the price of parts equal to zero, solve this problem in a natural way.

1 one of this matters, of course, unless open-source methods can actually deliver plentiful, highquality parts. After all, it is still better to have an expensive part than no part at all. So how confident are we that open-source incentives are up to the task?

We know from experience with the software industry that individual volunteers can be persuaded to write open-source code for various reasons, including altruism, education and the chance to impress others. Conversely, many synthetic biologists point out that creating and developing parts is far more expensive than writing code. For this reason, a practical open-parts collaboration will normally have to attract corporations as well as individuals. Here, the list of possible incentives is much shorter. We focus on two of the most important ones: the opportunity to share R\&D costs among multiple firms, and the opportunity to produce products faster through shared insights.

Even in this narrowed form, deciding when a corporation will make a dollarsand-cents decision to share R\&D is a difficult problem. Fortunately, synthetic biology is simpler than most industries. Most observers think that the emerging synthetic biology industry will, like the biotechnology industry (DiMasi \& Paquett, 2004), evolve into a series of niche product markets, each of which will ultimately be occupied by one-and only one-company. If so, there are only two possibilities. Consider first the case in which there are more markets than companies. Here, each firm targets a different market and no two companies ever compete. In this simple world, sharing poses no threat and drives down everyone's costs. Unfortunately, synthetic biologists do not live in this world. If they did, companies such as Amyris would not guard their data so closely and parts sharing would exist already.

Consider, then, the second case in which there are more companies than markets. Now the first company to deliver its product to a particular market is rewarded and its competitors will get nothing. This makes the decision to share a lot more difficult. Although sharing still reduces costs, it also increases the chances that some other company will win the race. In particular, we can no longer be sure that the usual opensource rule-'share everything immediately' —will attract enough companies to make sharing worthwhile. It is therefore reasonable to ask whether some other rule that makes sharing less costly-for example 'share everything after six months' or 'share everything after nine months' — would work better. Interestingly, something similar to this already happens in the open-source collaborations that produce 'embedded LINUX' software for mobile devices such as cell phones and DVD players. Here, member companies routinely interpret the GNU license so that they are not required to share 


\section{Sidebar A | What an open-parts license should look like}

For reasons explained in the text, a license would probably include the following clauses:

Goals. Each collaboration shares data on experiments that include a specific part. Members can pick and choose which collaborations to join.

Membership. Any company or individual can join the collaboration at any time. Furthermore, there are no membership fees. Members must, however, provide three months' notice before leaving. This prevents companies from 'taking a peek' at the collaboration's data and then immediately leaving to launch their own proprietary R\&D programmes.

Minimum sharing obligations. Members must share the results of all experiments, literature searches and computer simulations that include the collaboration's parts each time they (i) patent, sell, or otherwise make public any organism that contains the parts, (ii) abandon any project to develop such an organism or (iii) leave the collaboration.

Voluntary sharing. As with embedded LINUX, members will often find it in their interest to share data more often than the license requires. Game theory suggests that sharing can be encouraged by designing Collaboration facilities so that each member's data donations are maximally visible to everyone else (Maurer \& Scotchmer, 2006).

Making data public. The collaboration should let members make their data public at any time. Probably the simplest way is for members who want to keep information as a trade secret within the collaboration to attach a 'members only' designation to each submission. They could then remove this designation at any time. Disclosures that did not have the designation would be immediately posted on the collaboration's public website.

Intellectual property rights. The collaboration should allow members to seek patent and other intellectual property rights on any products that they develop containing the collaboration's parts. They would, however, waive these rights against members who used the patent's teachings to create organisms that (i) performed a substantially different function or (ii) performed the same function using a substantially different design.

Miscellaneous. Conventional open-source licenses often contain disclaimers and hold harmless clauses to keep volunteers from being sued if their code is defective. An open-parts agreement would probably contain similar terms.

code until their devices reach consumersin practice, a period of 18 months or so (Henkel, 2006).

$\mathrm{H}$ owever, this only restates the mystery: should a well-designed open-parts collaboration demand sharing after 18 months or 7 weeks or zero minutes? In general, this is a hard question. Indeed, trying to pick a particular number is a lot like guessing next week's closing price on Wall Street-possible within broad limits, perhaps, but perilous. Fortunately, the analysis for niche markets is significantly easier. Even if we cannot pick a specific period, we know that sharing will become costless as soon as the race has been won or lost. This suggests that a parts collaboration should at least require members to share as soon as the race ends - that is, when they patent an organism or offer it for sale or, less optimistically, abandon a project.

Moreover, this is only a minimum obligation as companies can still share sooner if they want to. This turns out to be important. In the software world, companies disclose roughly half of all embedded LINUX code before they have to (Henkel, 2006). The great advantage of this approach, of course, is that it allows those who understand the sharing problem best to set the sharing rule. Although this result is not perfect-there is a large economics literature on how gamesmanship and mistrust slow down otherwise logical sharing (Maurer \& Scotchmer, 2006)—-the result will almost certainly be more accurate than any top-down rule. Similar arguments would apply for any market in which the first company to produce a product obtains strong 'first mover advantages'.

S o much for theory. What would a collaboration look like in practice? The starting point, of course, would be to decide the scope of sharing. Probably the simplest and clearest approach would be to define collaboration by the gene sequences and homologies for a single part or small constellation of parts. The sharing obligation would then extend to any experiment that involved these DNA sequences whether or not other parts were involved.

Beyond this simple obligation, members would also need to interact, and this would require both physical and legal infrastructure. The physical component would probably consist of a password-protected online database where members could deposit and share data. Ideally, this facility would be flexible enough to include many different forms of data, including experimental test results, literature searches and computer simulations. As synthetic biologists have yet to agree on a standard nomenclature for parts, designing such a database is bound to be controversial. At the same time, collaboration organizers would have a clear incentive to please-and listen to-as many potential members as possible. There are surely worse ways to design a nomenclature.

The collaboration would also need a legal infrastructure. This would almost certainly consist of a license specifying the rights and duties of the members (Sidebar A). Here, the main legal problem is that gene data-unlike softwarecannot be copyrighted. Traditionally, most commentators have argued that patents can fill this gap. However, obtaining a patent is expensive-approximately $\$ 10,000$ in the US and $\$ 65,000$ in the European Union (Lawrence, 2008)-and at least one highly publicized attempt to build a patent-based 'protected commons' for plant data has produced negligible sharing (Jefferson, 2006).

$\mathrm{F}$ ortunately, patents are not the only option. Practically all jurisdictions also enforce agreements to protect commercially valuable trade secrets. As this protection only lasts until the information becomes public, trade secrets are significantly weaker than patents or copyright. However, this does not mean that trade secret protection is inferior. After all, an ideal open-parts license should interfere with users as little as possible. Provided that trade secrecy can be made to work, its weakness is actually desirable. That said, the weakness of trade secrecy does raise three design issues, all of which seem to be manageable.

First, secrecy can be lost through reverseengineering. For synthetic biology, this suggests that the DNA sequence or blueprint of a new organism would become public soon after it appeared on the market. Conversely, trade secrecy would continue for data that could not be reverse-engineered from the final product; for example, the design rules or dead-ends encountered along the way. Trade secrecy would also be preserved for 
organisms used in manufacturing processes conducted in private facilities that are closed to the public. Finally, companies that want to protect the DNA sequence of a new organism can still apply for patents. A well-designed consortium should allow patenting subject to a royalty-free cross-license for members.

Second, it is reasonable to think that third parties will sometimes independently discover and even patent the collaboration's secrets. In principle, this poses a nightmare scenario in which members must pay for the right to use their own inventions. In practice, firms can manage this risk by disclosing their inventions to the publicso-called 'defensive publishing' — so that no one can patent them. Members who share information with the collaboration should be allowed to make it public at any time.

The third problem with trade secrets is that-unlike patents or copyright-a single unauthorized disclosure can destroy protection. Can the collaboration really trust every last member to keep a secret? Companies and even academic laboratories can usually be trusted because secrecy gives them an advantage over competitors. But what about small actors such as students or hobbyists who might reveal collaboration data for reasons of ideology or malice? At least for now, excluding these small individuals from the collaboration seems unnecessarily harsh. Collaborations should first experiment with softer measures-community norms, expulsion of violators and/or threats of legal action.

$\mathrm{F}$ inally, an open-parts collaborationlike all agreements between competitors-will almost certainly invite scrutiny from antitrust authorities. Since the open-parts agenda is designed to block monopoly, sensible regulators should be indulgent. Nevertheless, reasonable issues do exist and will need to be managed. Probably the most obvious has to do with who can join the collaboration. In recent years, there has been an increasing tendency to apply the open-source label to any site where sharing occurs, including those that screen new members or, somewhat more subtly, charge a substantial fee to join. In economic terms, however, such arrangements are indistinguishable from patent pools and should draw the same scrutiny. This is probably not fatal, as patent pools often pass muster under the antitrust laws (US Supreme Court, 1947). Conversely, it would be even simpler to allow new members to join without any fees or preconditions. That would go a long way toward making the collaboration antitrust-compliant.

\section{More generally, the value of a part depends not only on its intrinsic cleverness, but also on how many times it has been used before}

There is also a deeper issue. The patent system is designed to promote races between inventors. Allowing synthetic biology companies to share parts will moderate this behaviour. In the extreme case in which every competitor joined the collaboration, racing would stop entirely. From a policy standpoint, this might or might not be desirable-although races deliver new parts sooner, they also generate duplication and waste. Once again, the fact that patent pools have similar effects and are routinely upheld in court suggests that the collaboration would survive such challenges. Finally, authorities often worry that even harmless collaborations can be used as 'sham agreements' to hide and facilitate illegal conspiracies. Here, the collaboration would have a good defence: provided that its transactions are truly transparent, the best answer to suspicious authorities might be: "Go ahead, see for yourself".

A s already mentioned, synthetic biology is a tipping market and Amyris' closed-parts business model has jumped out to an early lead. The US Government has been at least weakly complicit: The Department of Energy's \$350 million biofuels initiative has no open-parts requirement at all. The fact that commercial synthetic biology companies routinely receive government support makes this laissez faire attitude especially striking. When Bill Gates cornered the software market, he at least used private money. Pessimists will say that it is now too late for open-parts collaboration; optimists will reply that no one can possibly know this and that the only reasonable course is to act immediately.

The good news is that the open-parts idea has a long list of powerful friends. First, there are start-up companies. For every frontrunner such as Amyris, there are probably several would-be rivals who need shared open parts to catch up. Second, there are the gene synthesis companies that make synthetic DNA.
Demand for their products can only go up if parts designs cost nothing. Indeed, at least one gene synthesis company, Ginkgo Bioworks (Cambridge, MA, USA), has reportedly written open parts into its business plan. Third, there are the academics. Almost all of them will be better off if parts are cheap and open. Finally, there is government. Many jurisdictions already promote open source as a hedge against the Microsoft monopoly. The case for open parts is more or less identical.

One thing is clear: there has been nearly a decade of talk about open parts. At this point, the only way for synthetic biologists to learn more is to experiment with an actual collaboration. By the standards of synthetic biology, the cost of building the IT and legal infrastructure for a pilot-scale collaboration is small. Conversely, the potential upside is large. Tipping markets are notoriously imitative. If synthetic biologists can establish just one successful open-parts collaboration, industry will stand in line to build more.

\section{CONFLICT OF INTEREST}

The author declares that they have no conflict of interest.

\section{REFERENCES}

Cohn D (2005) Open-source biology evolves. Wired, January 17

DiMasi J, Paquett C (2004) The economics of follow-on drug research and development. Pharmacoeconomics 22 (Suppl 2): 1-14 Henkel J (2006) Selective revealing in open innovation processes: the case of embedded LINUX. Res Policy 35: 953-969

Henkel J, Maurer S (2007) The economics of synthetic biology. Mol Syst Bio/ 3:117

Jefferson R (2006) Science as social enterprise: the Cambia Bios initiative. Innovation 4: 13-44

Lawrence S (2008) Biotech patents: business as usual? Nat Biotechnol 26: 1326

Maurer S, Scotchmer S (2006) Open-source software: the new intellectual property paradigm. In Handbook on Information Systems, T Hendershott (Ed), pp 285-322. Amsterdam, the Netherlands: Elsevier

US Supreme Court (1947) Transwrap Machine Corporation vs. Stokes \& Smith Co. 329 U.S. 637

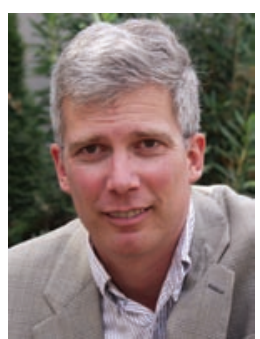

Stephen M. Maurer is at the Goldman School of Public Policy \& Berkeley Law School, University of California at Berkeley, USA. E-mail: smaurer@berkeley.edu doi:10.1038/embor.2009.165 